\title{
A note on the numerical simulations of flow past a wavy square-section cylinder
}

\author{
G. C. Ling - L. M. Lin
}

Received: 30 August 2007 / Accepted: 19 October 2007 / Published online: 8 January 2008

(C) Springer-Verlag 2007

\begin{abstract}
The flow past a square-section cylinder with a geometric disturbance is investigated by numerical simulations. The extra terms, due to the introduction of mapping transformation simulating the effect of disturbance into the transformed Navier-Stokes equations, are correctly derived, and the incorrect ones in the previous literature are pointed out and analyzed. Furthermore, the relationship between the vorticity, especially on the cylinder surface, and the disturbance is derived and explained theoretically. The computations are performed at two Reynolds numbers of 100 and 180 and three amplitudes of waviness of $0.006,0.025$ and 0.167 with another aim to explore the effects of different Reynolds numbers and disturbance on the vortex dynamics in the wake and forces on the body. Numerical results have shown that, at the mild waviness of 0.025 , the Kármán vortex shedding is suppressed completely for $R e=100$, while the forced vortex dislocation is appeared in the near wake at the Reynolds number of 180 . The drag reduction is up to $21.6 \%$ at $R e=100$ and $25.7 \%$ at $R e=180$ for the high waviness of 0.167 compared with the non-wavy cylinder. The lift and the Strouhal number varied with different Reynolds numbers and the wave steepness are also obtained.
\end{abstract}

Keywords Numerical simulation - Geometric disturbance . Square-section cylinder · Wake flow

The project supported by the National High-Tech Research and Development Program of China (863 Program) (2006AA09Z350) and the Knowledge Innovation Program of the Chinese Academy of Sciences (KJCX2-YW-L02).

G. C. Ling $(\varangle) \cdot$ L. M. Lin

The State Key Laboratory of Nonlinear Mechanics, Institute of Mechanics, Chinese Academy of Sciences, Beijing 100080, China

e-mail: linggc@imech.ac.cn

\section{Introduction}

Recently, the flow around bluff bodies with a geometric disturbance has been investigated by some experiments [1-3] and numerical simulations [4]. Previous results have shown that such three-dimensional geometric disturbance can interfere with the flow at upper/lower free shear layers and the regular vortex shedding, even leading to the complete suppression of Kármán vortex. And, cylinder drags, as well as amplitude of lifts, are reduced due to the disturbance. However, the physical mechanics responsible for such phenomena is not well understood. Devoting to understanding the matter by numerical simulations is very few. To the best of our knowledge, the newly published paper [4] could be the first numerical attempt at investigating the flow past the wavy cylinder with square section. Unfortunately, we found there were some fundamental errors in their mathematical formulation of the governing equations used for DNS. Particularly, as the mapping transformation is applied, the extra terms in the transformed $\mathrm{N}-\mathrm{S}$ equations are incorrectly presented, which was testified by authors in 2002 and confirmed by Sherwin in 2003 in a private communication. Now the aim of the note is to present the right mathematical formula used for DNS and particularly to give the extra terms in transformed equations. The expression of vorticity on the cylinder surfaces is given for better understanding the relationship between the vorticity and the waviness, which is of importance in the redistribution of streamwise and vertical vorticities near the cylinder and in the near wake. The effects of various geometric disturbance and the different Reynolds numbers on the vortex dynamics in the wake and the forces on the body are further investigated numerically. On the basis of DNS results, the vortex structures and the variations of forces are presented briefly at $R e=100$ and 180 . 

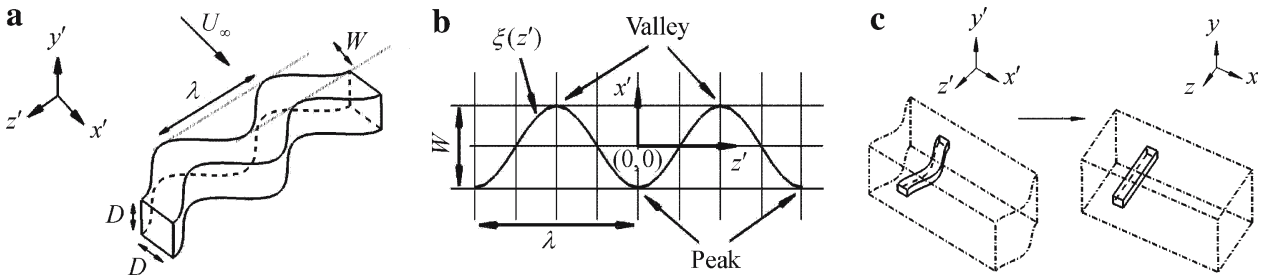

Fig. 1 a Diagram of uniform flow past the wavy square-section cylinder; b Schematic of harmonic disturbance, $\xi\left(z^{\prime}\right)$, in the plane of $\left(x^{\prime}, z^{\prime}\right)$; c Mapping transformation from the physical flow field $\left(x^{\prime}, y^{\prime}, z^{\prime}\right)$ to the computational domain $(x, y, z)$

\section{Transformation of Navier-Stokes equations and vorticity on cylinder surfaces}

For flows past a wavy square-section cylinder in an original cartesian coordinate system $\left(x^{\prime}, y^{\prime}, z^{\prime}\right)$, as shown in Fig. 1a, the imposed harmonic waviness limited in the $\left(x^{\prime}, z^{\prime}\right)$ plane in Fig. $1 \mathrm{~b}$ can be expressed mathematically by:

$\xi\left(z^{\prime}\right)=-\frac{W}{2} \cos \left(\frac{2 \pi}{\lambda} z^{\prime}\right)$,

where $W$ is the wave height and $\lambda$ the spanwise wavelength. It is of convenience in the mesh generation by applying the simplified mapping, which was suggested by Newman [5] in describing the freely vibrating cables. Therefore, the physical flow field with the deformed body is transformed into the computational domain with the straight one in the transformed reference frame $(x, y, z)$ (see Fig. 1c). The mapping transformation is then defined as:

$x=x^{\prime}-\xi\left(z^{\prime}\right), \quad y=y^{\prime}, \quad z=z^{\prime}$.

The incompressible dimensionless continuity and NavierStokes equations are written in the original coordinate system $\left(x^{\prime}, y^{\prime}, z^{\prime}\right)$ as

$\nabla^{\prime} \cdot \boldsymbol{u}^{\prime}=0$

$\frac{\partial \boldsymbol{u}^{\prime}}{\partial t}+\left(\boldsymbol{u}^{\prime} \cdot \nabla^{\prime}\right) \boldsymbol{u}^{\prime}=-\nabla^{\prime} p^{\prime}+\frac{1}{R e} \nabla^{\prime 2} \boldsymbol{u}^{\prime}$,

where $t$ is the time, $\boldsymbol{u}^{\prime}=\left(u^{\prime}, v^{\prime}, w^{\prime}\right)$ is the velocity vector, $p^{\prime}$ is the static pressure, $R e$ is the Reynolds number defined as $U_{\infty} D / v$, where $v$ is the kinematic viscosity of the fluid, and $\nabla^{\prime}$ is the gradient operator. All lengths are scaled by the cylinder base height $D$ and velocities by the free-stream velocity $U_{\infty}$. Furthermore, the relationship of derivatives is modified only in $z^{\prime}$ component as following,

$\frac{\partial}{\partial x^{\prime}}=\frac{\partial}{\partial x}$,

$\frac{\partial}{\partial y^{\prime}}=\frac{\partial}{\partial y}$,

$\frac{\partial}{\partial z^{\prime}}=\frac{\partial}{\partial z}-\frac{\mathrm{d} \xi}{\mathrm{d} z} \frac{\partial}{\partial x}$, where the slope of the waviness is given by:

$\frac{\mathrm{d} \xi}{\mathrm{d} z}=\frac{\mathrm{d} \xi}{\mathrm{d} z^{\prime}}=\pi \frac{W}{\lambda} \sin (\beta z), \quad$ with $\left|\frac{\mathrm{d} \xi}{\mathrm{d} z}\right|<1$,

where $\beta=2 \pi / \lambda$ is the wave number, the ratio of $W$ to $\lambda$ is defined as the wave steepness, becoming the important parameter in the flow control and determining the strength of disturbance.

Differentiating Eq. (4) and applying the chain rule, the velocities and pressure are then transformed by the following form:

$u=u^{\prime}-w^{\prime} \mathrm{d} \xi / \mathrm{d} z, \quad v=v^{\prime}, \quad w=w^{\prime}, \quad p=p^{\prime}$.

In the transformed coordinate system $(x, y, z)$, the continuity and Navier-Stokes equations $(3 \mathrm{a}, \mathrm{b})$ are derived carefully and obtained as:

$$
\begin{aligned}
& \nabla \cdot \boldsymbol{u}=0, \\
& \begin{aligned}
\frac{\mathrm{D} \boldsymbol{u}}{\mathrm{D} t} & =\frac{\partial \boldsymbol{u}}{\partial t}+(\boldsymbol{u} \cdot \nabla) \boldsymbol{u} \\
& =-\nabla p+\frac{1}{\operatorname{Re}} \nabla^{2} \boldsymbol{u}+\boldsymbol{A}(\boldsymbol{u}, p, \xi ; R e),
\end{aligned}
\end{aligned}
$$

where $\boldsymbol{A}$ is the extra term introduced by the non-inertial transformation (2) indicating the effect of disturbance on the fluid dynamics. The right expressions of three components of $\boldsymbol{A}$ should be

$$
\begin{aligned}
A_{x}= & -\left[\frac{\mathrm{d}^{2} \xi}{\mathrm{d} z^{2}} w^{2}+\left(\frac{\mathrm{d} \xi}{\mathrm{d} z}\right)^{2} \frac{\partial p}{\partial x}-\frac{\mathrm{d} \xi}{\mathrm{d} z} \frac{\partial p}{\partial z}\right] \\
& +\frac{1}{\operatorname{Re}}\left[-2 \frac{\mathrm{d} \xi}{\mathrm{d} z} \frac{\partial^{2} u}{\partial x \partial z}+\left(\frac{\mathrm{d} \xi}{\mathrm{d} z}\right)^{2} \frac{\partial^{2} u}{\partial x^{2}}\right. \\
& \left.+\frac{\mathrm{d}^{2} \xi}{\mathrm{d} z^{2}}\left(2 \frac{\partial w}{\partial z}-\frac{\partial u}{\partial x}\right)-2 \frac{\mathrm{d} \xi}{\mathrm{d} z} \frac{\mathrm{d}^{2} \xi}{\mathrm{d} z^{2}} \frac{\partial w}{\partial x}+\frac{\mathrm{d}^{3} \xi}{\mathrm{d} z^{3}} w\right], \\
A_{y}= & \frac{1}{\operatorname{Re}}\left[\frac{\partial^{2} v}{\partial z^{\prime 2}}-\frac{\partial^{2} v}{\partial z^{2}}\right], \\
A_{z}= & \frac{\mathrm{d} \xi}{\mathrm{d} z} \frac{\partial p}{\partial x}+\frac{1}{\operatorname{Re}}\left[\frac{\partial^{2} w}{\partial z^{\prime 2}}-\frac{\partial^{2} w}{\partial z^{2}}\right] .
\end{aligned}
$$


Fig. 2 Schematics of $\mathbf{a} w$ and $\xi(z)$, and $\mathbf{b} \partial^{2} w / \partial z^{2}$ and $\mathrm{d}^{2} \xi / \mathrm{d} z^{2}$ varying in the spanwise direction, where magnitudes of amplitudes are not real values. The dashed line denotes zero value
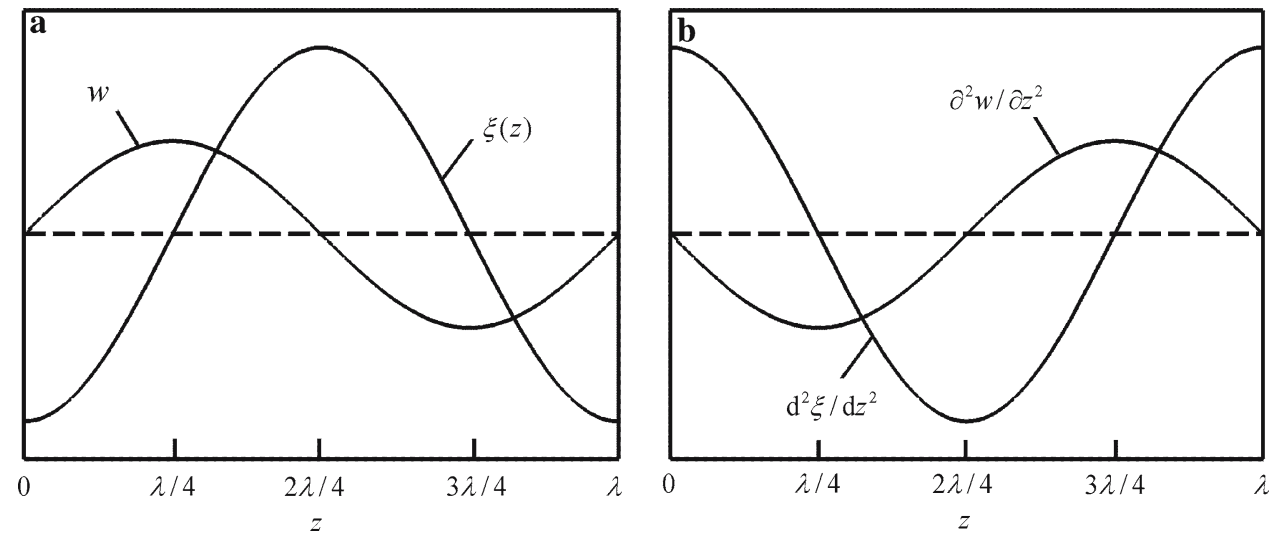

Compared with results obtained in Darekar and Sherwin [4], errors are found out in viscous parts in $x$ and $z$ components of the extra terms which are listed as

$$
\begin{aligned}
A_{x \text { (viscous) }}= & \frac{1}{R e}\left[-2 \frac{\mathrm{d} \xi}{\mathrm{d} z} \frac{\partial^{2} u}{\partial x \partial z}+\left(\frac{\mathrm{d} \xi}{\mathrm{d} z}\right)^{2} \frac{\partial^{2} u}{\partial x^{2}}\right. \\
& \left.-\frac{\mathrm{d} \xi}{\underline{\mathrm{d} z} \frac{\partial^{2} w}{\partial z^{2}}}-\frac{\mathrm{d}^{2} \xi}{\mathrm{d} z^{2}} \frac{\partial u}{\partial x}+\frac{\mathrm{d} \xi}{\mathrm{d} z} \frac{\mathrm{d}^{2} \xi}{\mathrm{d} z^{2}}+\frac{\mathrm{d}^{3} \xi}{\mathrm{d} z^{3}} w\right], \\
A_{z \text { (viscous) }}= & \frac{1}{R e}\left[\frac{\partial^{2} w}{\partial z^{\prime 2}}-\frac{\mathrm{d}^{2} \xi}{\underline{\mathrm{d} z^{2}}}\right],
\end{aligned}
$$

where the wrong terms are marked by the underline. From the point of physical view, there should be the strain in the gradient of viscous stress in viscous parts generally, such as $\partial w / \partial z$ in $(1 / R e)\left(\partial^{2} w / \partial z^{2}\right)$ in Eq. (8c), rather than the slope of wavy disturbance $\mathrm{d} \xi / \mathrm{d} z$ in Eq. (9b). By means of dimension analysis, we found that there is a lack of dimension of time in the wrong expressions $(\mathrm{d} \xi / \mathrm{d} z)\left(\mathrm{d}^{2} \xi / \mathrm{d} z^{2}\right)$ and $\mathrm{d}^{2} \xi / \mathrm{d} z^{2}$ in Eqs. (9a) and (9b), respectively, compared with other terms. Furthermore, according to the flow near the front of cylinder obtained in our numerical simulations, the spanwise velocity $w\left(=w^{\prime}\right)$ varies with the extremum near the inflexion $(\xi=0)$ and zero at peaks and valleys, respectively, proportional to $\sin (\beta z)$ shown in Fig. 2a. Then there are $\partial^{2} w / \partial z^{2} \propto-\sin (\beta z)$ and $\mathrm{d}^{2} \xi / \mathrm{d} z^{2} \propto \cos (\beta z)$, as shown in Fig. 2b. We can see that the contribution of non-zero behavior of $\partial^{2} w / \partial z^{2}$ in Eq. (8c) near the inflexion to the spanwise acceleration $\mathrm{D} w / \mathrm{D} t$ in the $z$ component of Eq. (7b), rather than no influence of $\mathrm{d}^{2} \xi / \mathrm{d} z^{2}(\approx 0)$ in Eq. (9b). Similar analyses will be taken at peaks and valleys. Obviously, the incorrect spanwise extra term will lead to the distortion of distribution of $w$, then affect the streamwise velocity through the coupling of pressure gradients between the streamwise and spanwise flows of Eqs. (8a) and (8c). Such perturbed flow induces the surface vorticity resulting in the distorted shear flow near the cylinder and near wake.
Therefore, results of flow patterns and force variations obtained in Darekar and Sherwin [4] are suspicious.

We are also interested in the distribution of vorticity field and the relationship with the disturbance. The transformation of vorticity fields between two coordinate systems is performed as

$\omega_{x}^{\prime}=\omega_{x}+\frac{\mathrm{d} \xi}{\mathrm{d} z} \frac{\partial v}{\partial x}$

$\omega_{y}^{\prime}=\omega_{y}+\frac{\mathrm{d} \xi}{\mathrm{d} z}\left(\frac{\partial w}{\partial z}-\frac{\partial u}{\partial x}\right)-\left(\frac{\mathrm{d} \xi}{\mathrm{d} z}\right)^{2} \frac{\partial w}{\partial x}+\frac{\mathrm{d}^{2} \xi}{\mathrm{d} z^{2}} w$

$\omega_{z}^{\prime}=\omega_{z}-\frac{\mathrm{d} \xi}{\mathrm{d} z} \frac{\partial w}{\partial y}$.

Specially, the distribution of vorticity at surfaces is simplified due to the nonslip condition $\boldsymbol{u}^{\prime}=\boldsymbol{u}=\mathbf{0}$ as following:

$\left.\omega_{x}^{\prime}\right|_{ \pm x}=\left.\frac{\mathrm{d} \xi}{\mathrm{d} z} \omega_{z}\right|_{ \pm x}$

$\left.\omega_{x}^{\prime}\right|_{ \pm y}=\left.\omega_{x}\right|_{ \pm y}$

$\left.\omega_{y}{ }^{\prime}\right|_{ \pm x}=\left.\omega_{y}\right|_{ \pm x}+\left.\left(\frac{\mathrm{d} \xi}{\mathrm{d} z}\right)^{2} \omega_{y}\right|_{ \pm x}$,

$\left.\omega_{y}^{\prime}\right|_{ \pm y}=\left.\omega_{y}\right|_{ \pm y} \equiv 0$

$\left.\omega_{z}^{\prime}\right|_{ \pm x}=\left.\omega_{z}\right|_{ \pm x}$,

$\left.\omega_{z}{ }^{\prime}\right|_{ \pm y}=\left.\omega_{z}\right|_{ \pm y}-\left.\frac{\mathrm{d} \xi}{\mathrm{d} z} \omega_{x}\right|_{ \pm y}$,

where the subscript $\pm x$ and $\pm y$ denote the front/back, and upper/lower sides of cylinder, respectively. Equations (11) show that the vorticity is strongly dependent on the wave steepness. The streamwise and vertical components of vorticity are coupled each other. The mechanics of generating vertical vorticity on the upper/lower surfaces is identical with that in the straight cylinder because of the disturbance limited in $\left(x^{\prime}, z^{\prime}\right)$ plane. From another point of view, the surface vorticities can be reformulated by 

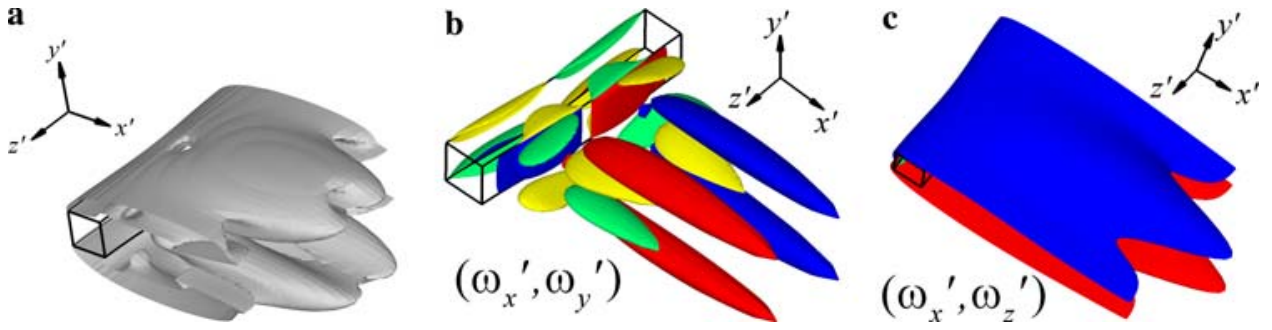

Fig. 3 Vortex patterns: a $\lambda_{2}$ description, $\mathbf{b}$ the iso-vorticity surfaces of $\omega_{x}{ }^{\prime}$ and $\omega_{y}{ }^{\prime}$, and $\mathbf{c}$ the iso-vorticity surfaces of $\omega_{x}{ }^{\prime}$ and $\omega_{z}{ }^{\prime}$ at $t=480$, $R e=100$ and $W / \lambda=0.025$

$$
\begin{aligned}
& \left.\frac{\omega_{x}{ }^{\prime}}{\omega_{z}{ }^{\prime}}\right|_{ \pm x}=\frac{\mathrm{d} \xi}{\mathrm{d} z} \propto \frac{W}{\lambda}, \\
& \text { if }\left.\omega_{z}{ }^{\prime}\right|_{ \pm x}=\left.\frac{\partial v}{\partial x}\right|_{ \pm x} \neq 0 \\
& \left.\omega_{y}^{\prime}\right|_{ \pm x}=\left[1+\left(\frac{\mathrm{d} \xi}{\mathrm{d} z}\right)^{2}\right]\left(-\left.\frac{\partial w}{\partial x}\right|_{ \pm x}\right), \\
& \left.\frac{\omega_{z}^{\prime}-\omega_{z}}{\omega_{x}{ }^{\prime}}\right|_{ \pm y}=\frac{\mathrm{d} \xi}{\mathrm{d} z} \propto \frac{W}{\lambda}, \\
& \text { if }\left.\omega_{x}^{\prime}\right|_{ \pm y}=\left.\frac{\partial w}{\partial y}\right|_{ \pm y} \neq 0 .
\end{aligned}
$$

On the front/back surfaces, the streamwise vorticity is proportional to the spanwise vorticity, as well as the wave steepness with the linear variation in Eq. (12a), while the vertical vorticity is generated as a result of the variation of spanwise velocity induced by the streamwise vorticity and related with the wave steepness in nonlinear variations in Eq. (12b). On the basis of the above formula, we can understand how the geometric disturbance influences the vorticity distribution on the cylinder surfaces, further convecting into the near wake.

\section{Numerical result}

By utilizing the hybrid method of Fourier spectral and the finite difference with multigrid Poisson solver, the flow around the cylinder at $R e=100$ and 180 and $\lambda=5.6$ with different wave steepness are numerically performed. The flow is assumed to be periodic in the spanwise direction. In the $(x, y)$-plane, the boundary conditions are prescribed as the following: (a) the free stream velocities $U_{\infty}=1, v=$ $w=0$ at the inlet; (b) the convective boundary condition $\partial \boldsymbol{u} / \partial t+U_{c}(\partial \boldsymbol{u} / \partial x)=0$ with $U_{c}=1$ at the outlet; (c) noslip conditions on the cylinder surfaces; and (d) free-slip or frictionless wall conditions $\partial u / \partial y=v=\partial w / \partial y=0$ at the upper and lower confining surfaces in the computational domain. Consistent boundary conditions for the normal gradient of pressure from Eq. (7b) are used, especially simplified as $\partial p / \partial n=0$ in the normal direction $n$ at inlet, outlet and upper/lower boundaries.
In this note, only some results for $W / \lambda=0.025$ at two Reynolds numbers are given. Large parts of evolution on vortex dynamics and features of strong effect of disturbance will be presented in the future paper for different wave steepnesses and Reynolds numbers. The wake topology described by different ways in Fig. 3 at $R e=100$ shows that the main vortex core is located at free shear layers and the near wake, denoted by the $\lambda_{2}$ description of -0.01 [6]. Such vortex structure is symmetric about the wake center, mainly consisting of streamwise and vertical vorticities in the near wake and the spanwise vorticity in free shear layers. Free shear layers are wavy, and the wake width is varied in the spanwise direction. The iso-surface of definition shows that the vortex is mainly located in the vicinity of the cylinder, called the "Chayote"-like vortex, followed by a steady vorticity layer. In the wake the alternating shedding of Kármán vortex is completely suppressed. The physical mechanics responsible for the phenomenon is one of main interesting objects in future. When the Reynolds number increases up to 180 , the wake dynamics indicates the strong competition between the natural secondary instability and the effect of disturbance. The vortex structure is surprisingly different from the one at $R e=100$ in the disappearance of "Chayote"-like vortex in the near wake and the emergence of Mode A-like vortex only in the little far wake, as shown in Fig. 4, where Mode $\mathrm{A}$ is induced by the secondary instability [7]. According to the distributions of the spanwise vorticity and pressure [8] in Fig. 4b and c, the vortex dislocation is identified, and the shear layers are farther elongated downstream than those in flows without the geometric disturbance. As the black arrow shown in Fig. 4b, dislocation is located at the valley.

The Strouhal number, $S t$, analyzed from the spanwise averaged lift coefficient, decreases once the disturbance is introduced, compared with that in flows around the non-wavy cylinder in Fig. 5a. In particular $S t=0$ appears in the case of "Chayote"-like vortex. We note that the force variation is mainly due to the three-dimensional shear flow near the cylinder resulted from the disturbance. Figures $5 \mathrm{~b}$ and $\mathrm{c}$ show that with the increase of wave steepness, the time-average drag reduction increases, even up to the $21.6 \%$ at $R e=100$ and $25.7 \%$ at $R e=180$ in the case of $W / \lambda=0.167$, different from the results of Darekar and Sherwin [4] from $15.9 \%$ at 

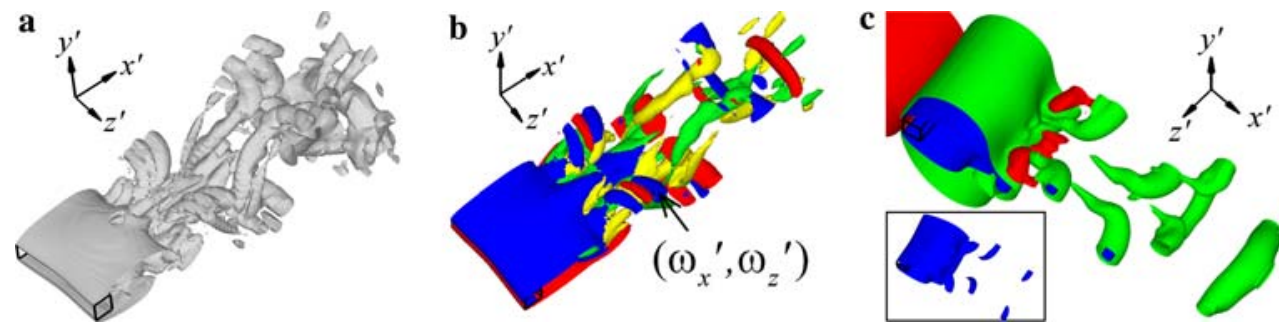

Fig. 4 Vortex patterns: a $\lambda_{2}$ description, $\mathbf{b}$ the iso-vorticity surfaces of $\omega_{x}{ }^{\prime}$ and $\omega_{z}{ }^{\prime}$, and $\mathbf{c}$ the iso-pressure surface at $t=340, R e=180$ and $W / \lambda=0.025$

Fig. 5 Parameters varying with the wave steepness and $R e$ a $S t$, b $\bar{C}_{D}$ and $\mathbf{c} C_{L}^{\prime}$

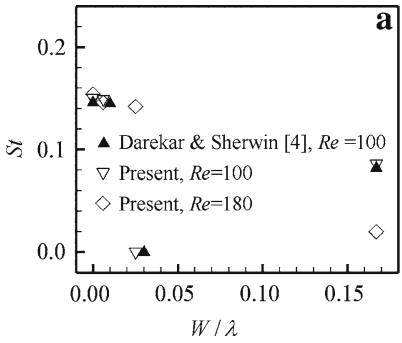

$W / \lambda=0.03$ to $15.1 \%$ at $W / \lambda=0.167$, which could be because of the incorrect shear flow near the cylinder resulting from Eqs. (9). While the RMS lift coefficient reaches to the minimum at $W / \lambda=0.025$.

\section{References}

1. Bearman, P.W., Tombazis, N.: The effects of three-dimensional imposed disturbances on bluff body near wake flows. J. Wind Eng. Indust. Aerodyn. 49, 339-350 (1993)

2. Tombazis, N., Bearman, P.W.: A study of three-dimensional aspects of vortex shedding from a bluff body with a mild geometric disturbance. J. Fluid Mech. 330, 85-112 (1997)
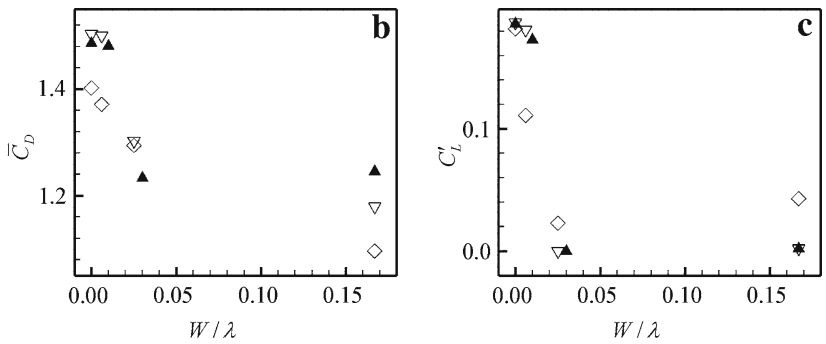

3. Bearman, P.W., Owen, J.C.: Reduction of bluff-body drag and suppression of vortex shedding by the introduction of wavy separation lines. J. Fluids Struct. 12, 123-130 (1998)

4. Darekar, R.M., Sherwin, S.J.: Flow past a square-section cylinder with a wavy stagnation face. J. Fluid Mech. 426, 263-295 (2001)

5. Newman, D.J.: A computational study of fluid/structure interactions: flow-induced vibrations of a flexible cable. Ph.D. thesis, Princeton University, pp. 9-13 (1996)

6. Jeong, J., Hussain, F.: On the identification of a vortex. J. Fluid Mech. 285, 69-94 (1995)

7. Robichaux, J., Balachandar, S., Vanka, S.P.: Three-dimensional Floquet instability of the wake of square cylinder. Phys. Fluids 11(3), 560-578 (1999)

8. Braza, M., Faghani, D., Persillon, H.: Successive stages and the role of natural vortex dislocations in three-dimensional wake transition. J. Fluid Mech. 439, 1-41 (2001) 